\title{
Velocity based Tracking and Localization System using Smartphones with GPS and GPRS/3G
}

\author{
Ibrahim Abdallah Hag Eltoum \\ Department of Computer Engineering \\ Laboratory of the Computer System and \\ Telecommunication "LCST" \\ FST, Abdelmalek Essaadi University, \\ Tanger, Morocco.
}

\author{
Mohammed Bouhorma \\ Department of Computer Engineering \\ Laboratory of the Computer System and \\ Telecommunication "LCST" \\ FST, Abdelmalek Essaadi University, \\ Tanger, Morocco.
}

\begin{abstract}
Smartphones are advanced versions of cell phones which can provide multi functions tasks. Most Smartphones have the following facilities: E-mails, Cameras, Wi-Fi connectivity, and comprehensive user interface such as touch screen and built in GPS system and interface for installing new applications. Using outdoor location-based services associated with GPS/GPRS have widely out broken. This paper aims at developing a velocity based system for location tracking that will be more convenient for users and more reliable, putting into consideration the expenses for the network service and increasing the accuracy in Smartphones.
\end{abstract}

\section{Keywords}

Tracking; Location System; GPS; GPRS; Smartphones; Android Application.

\section{INTRODUCTION}

Developments and progress in mobile computing have led to a wide range of services and functions available for users. The use of outdoor location-based services associated with GPS has become phenomenal. Today using outdoor location tracking and control systems is widely used, especially in Smartphones where a signal receiving devices has been installed.

Many applications are compatible to be used with GPS in Smartphones and the tracking system can also be used with a built-in system.

Nowadays the Global Positioning system (GPS) has been used on a large scale after it became free of charge for people, with the lower cost of the signal component, (GPS) has increasingly being used in our daily life in many fields such as [1] the steering system as in cars and ships, the safety system such as tacking system for objects and living things, for example caring for old people, children and disabled people. Also in management systems, traffic police investigations system, etc. However most of these systems are online that work with a server as storage for travelling data which depends on the GPRS System as a channel for communication.

This mean the service charge per month is not suitable for work that doesn't require location tracking all the time.

In order to reduce the expense the system must be used only when necessary or when there is an unusual case.
In online using for SMS sending the location data to the user is not convenient, because of the SMS limitation and the word length and the user must remember the instruction required for sending SMS. to the location tracking device and the expenses for operating must be put into consideration.

Location tracking is a popular system nowadays- the online system is usually used in large organization with many tracking devices and hence very high monthly expense. After considering real usage, it can be seen that tracking must not done all the time but only when something unusual happens or when the data is required so that expense will be less.

A number of research mentioned development of location tracking for instance. Kalman filter [2] is used to increase the accuracy of data received from GPS satellite and of sending tracking data through SMS. Other studies include improvement of a low-power tracking device [3], development of a system for tracking and searching lost mobile devices using SMS [4], design of a logistics system for transportation vehicles [5], based on using barcode technology, car monitoring, a letting and tracking model enhancement with mobility and database facilities [6] which reports to the police station if any accident through the use of a camera and MMS relay to a server.

This paper is organized as follows: Section 2 contains a detailed description of the GPS. In Section 3 this paper describes the Smartphone \& operating system used in it. Section 4 discusses the features of the tracking system. In Section 5 this paper highlights the system overview. In Section 6 the result is present. This paper is concluded in section 7 .

\section{GPS}

In addition to the sensors mentioned before, mobile phones readily support GPS. GPS is used to obtain the current location and is used in outdoor navigation. The location of the device is estimated from signals sent from satellites. The Global Positioning System (GPS) is a well known means of obtaining locations in outdoor areas. However, this technique cannot be utilized in indoor environments as buildings and tunnels and congested metropolitan areas because of its reliance on satellite visibility There have been many efforts made to equip the GPS for indoor environments, efforts, for example, involving augmentation with an inertial navigation system (INS) or ["pseudo-satellite"] system [7,8]. Also, many researchers have proposed alternate systems for indoor areas [9]. 
A GPS satellite sends a beacon containing its position and the time at which the beacon was sent. From these beacons the GPS receiver calculates its own position. For this, it needs to receive beacons from at least four satellites. In addition to this the quality of the signal depends on the receiver having a clear view of the sky. As a result the quality of GPS signals is degraded with the amount of obstruction around the receiver, rendering the system inapt for indoor navigation. The time needed until the first $\mathrm{x}$ is calculated, can be shortened by using assisted GPS (AGPS). An additional source is used to estimate a coarse location, for example on a Smartphone, through looking up the location of the current cell tower the phone is connected to. With this method the time needed for a first $\mathrm{x}$ can be shortened.

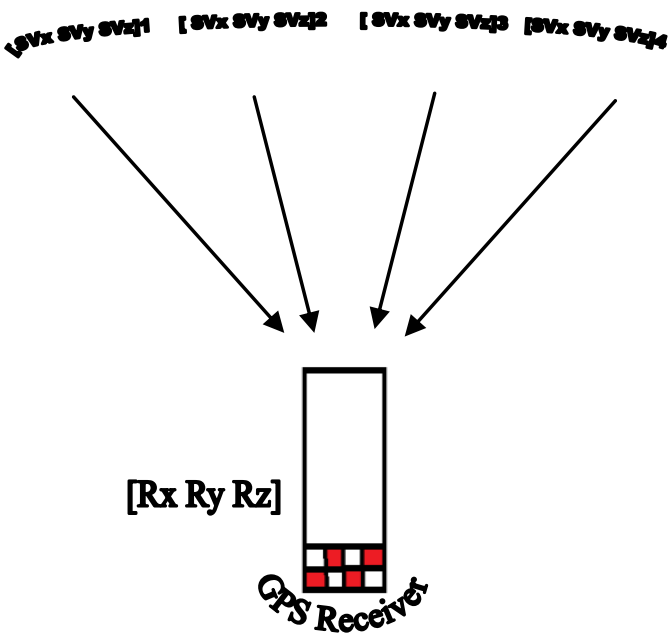

Fig 1: Line-of-sight pseudorange GPS measurements from at least four satellites

\section{SMARTPHONES}

Smartphones are considered as the new generation mobile devices. Day after day Smartphones becomes more popular. Smartphones run with the fundamental parts of software system. This operating system which has been designed for these smart devices should have sufficient energy with fewer memory footprint and more development and optimizations [8]. S. PH all and E Anderson [10].

By introducing the most common operating system in the market such as Blackberry, windows mobile, iPhone, Symbian and Android. So Smartphones depend on the mentioned operating systems and with the rapid and fast progress of Smartphones, international Smartphones shipments are expected to increase to reach 506 million units in 2014, from 246.9 million in 2010. And among these Smartphones, shipments of iPhone and Android have accelerated by launching HTC Hero and Nuxus and iPhone 3GS [11].

\subsection{Android:}

Android is the first development plat form and operating system for mobile phones that open, complete and free. Android was developed by the open Handset Alliance a group of over 30 companies led by Google when announcing Android, Google goal was to offer more flexible feature-rich platform in order to attract more community developers and use those advanced application to convince customer to buy Android handsets [12.13]. Android is considered as an asset of software for mobile devices which contain an operating system, middleware and main mobile applications. Android covers different features for instance: applications format framework, Dalvik Virtual machine, integrated browser, optimized graphics, SQL its for structured data storage, media support for common audio, video and image format, GSM technology, Bluetooth, EDG, 3G and Wi-Fi, Camera, GPS, compass and accelerometer and rich development environment [14. 15]. The architecture of Android is like any other hierarchical system, it is divided into four main layers that consist of:

\subsubsection{Application:}

Consist of asset of core application including email client, calendar, web browser, map application, SMS application, contact application, message application ... etc.

\subsubsection{Application framework:}

This is the base of developing application in Android and it has been designed to facilitate the reuse of components and allow the components to be replaced by users $[12,14,15]$.

\subsubsection{Libraries:}

They are set of $\mathrm{C} / \mathrm{C}++$ libraries used by diversity of components of Android system [12, 14, 15].

Android Runtime consist of core libraries and Dalvik virtual machine. Core libraries provide the functionalities which are available in Java programming language. Dalvik Virtual works as a translator between the application side and the operating system.

Every Android application operates in its own process and with its own instance of the Dalvik virtual machine [14, 15].

\subsubsection{Linux Kernel:}

Linux kernel performs as an abstraction layer between the hardware and Android software. Android uses Linux version 2.6 for core system services such as security, memory

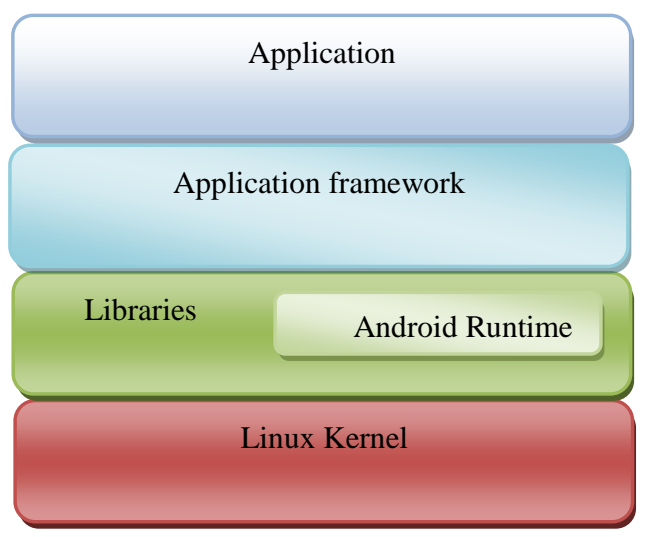

Fig 2: the architecture of Android

\section{FEATURES OF THE TRACKING SYSTEM:}

Generally the location tracking and determinate position can be categorized depends on the usage as follows:

$-\quad$ Real time online.
- $\quad$ Off line.

- $\quad$ Semi off line. 


\subsection{The Real Time online:}

The Real Time online device receives the real time position from the satellite and relays the information to the general online system is based on GPRS/EDGD/3G for the information relayed to the server.

This online tracking system common with operations requiring locations at all time for example: logistic system, traffic system taxi system, etc. The advantages appear in the convenience of use-Managing and controlling is also efficient due to the server which acts as a monitor and controller. However, 24 hour information relay results in high monthly expense from GPRS/EDGD/3G. Also incurring expense, or the server as the System Manager.

\subsection{The offline:}

Device also receives the present location from the satellite and records various data such as position, time, speed etc in its memory storage and it is the same as the black box of airplane. The information is not relayed to the user. The user must check recorded data when it is needed. So there is no expense because the system is connected to a network.

\subsection{The Semi-Offline:}

The Semi-Offline receives the real time location from the satellite and sends it to the user as requested. Sending data can be based on GPRS/EDGD/3G or form of SMS. The advantage of SMS Semi-offline is that SMS costs less than GPRS/EDGD/3G. The semi offline is thus using the tracking system at the time being [16].

\section{SYSTEM OVERVIEW}

The proposed tracking system in this paper is designed to track and monitor Smartphones, status that is used by certain party for particular purposes. This system is an integration of several modern embedded and communication technologies. To provide location and time information anywhere in the world, Global Positioning System (GPS) "built in the Smartphone" is commonly used as a space-based global navigation satellite system. The location information provided by GPS systems can be visualized using Google Earth. The path of this application can monitor the location graphically on Google Earth; it also can view other relevant information of each Smartphone in the fleet by using Real Time online method.

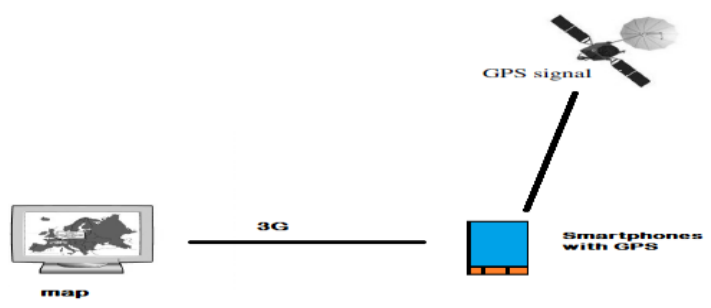

Fig 3: The architecture of system.

\subsection{The System model:}

The Distance: can be calculated using the well known formula to calculate the distance between two points as follows:

$$
D=\sqrt{\left(x_{2}-x_{1}\right)^{2}+\left(y_{2}-y_{1}\right)^{2}}
$$

The Velocity: calculate the velocity as follows:

$$
V=\frac{\Delta s}{\Delta t}
$$

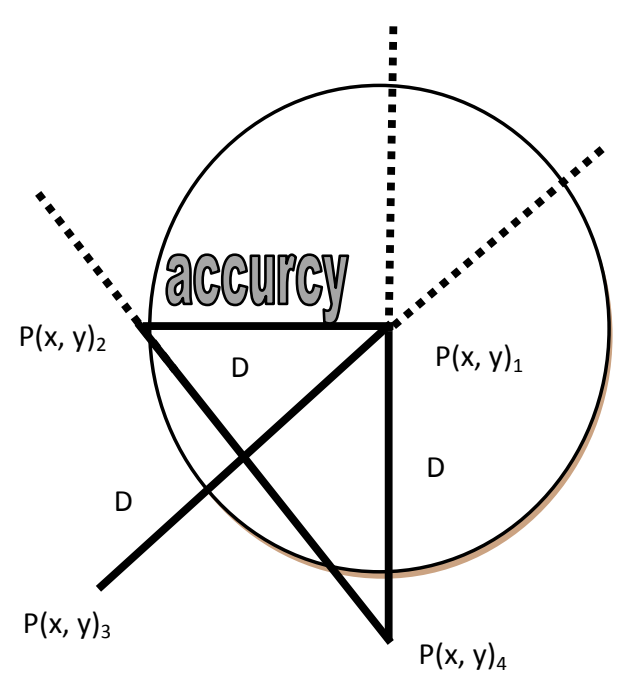

Fig 4: determine the line of tracking object.

\subsection{The velocity of the object:}

Our application corresponds with all the devices that support Android system in their different types.

This research found that the proposed application can show the different records which can be obtained to determine location in which the device exist. It collects the records form GPS and Maps with other records which can determine location and compare them to restore the most accurate ones.

In the case of no current records are available, it restore the previous point as a current point in order to continue in searching for new location on the contrary to the application that use GPS as they can't overcome the area which they couldn't find record for it next area.

Compared to the application that depends on GPS records, the proposed application obtained more accuracy.

And to achieve this, this research tried to find the velocity of object which to use it to determine its location. That is, if the application knows the current and previous point it can calculate the distance and the time of the object in its movement from the previous point to the current and through the equation (2), the proposed application can find the speed of the object. The steps of finding the velocity and positioning the objects are described in Figure 5. 

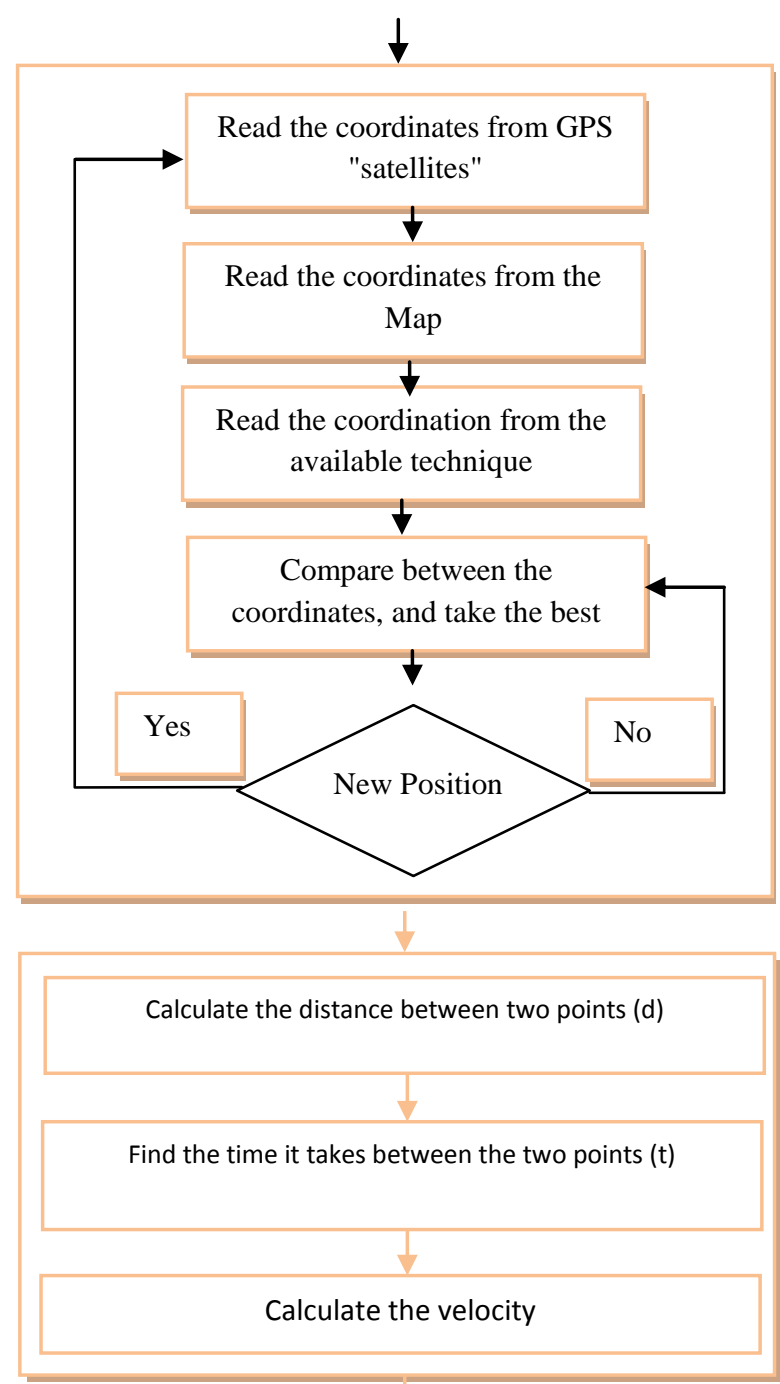

Fig 5: Calculating the Velocity.

\section{RESULT}

This research has developed the application so that to be reliable in determining the location. The accuracy exceeded 5 meters and as described in Figure 6.2 below it can be seen that the accuracy reached less than 5 meters and ranged between 5meters and even 2 meters. To increase the accuracy of determination of the location in the final point (the last point where GPS stopped), this research used the velocity equation to increase the accuracy of tracking " Figure 6.3", depending on the speed and slope of the line to determine the destination, which can be used everywhere.

(1)

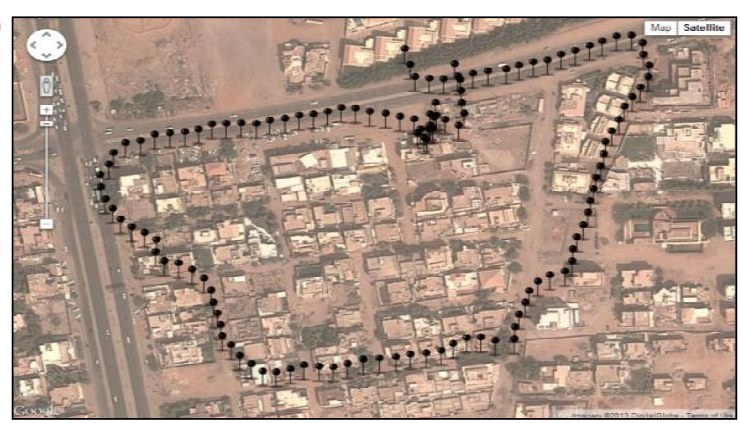

(2)

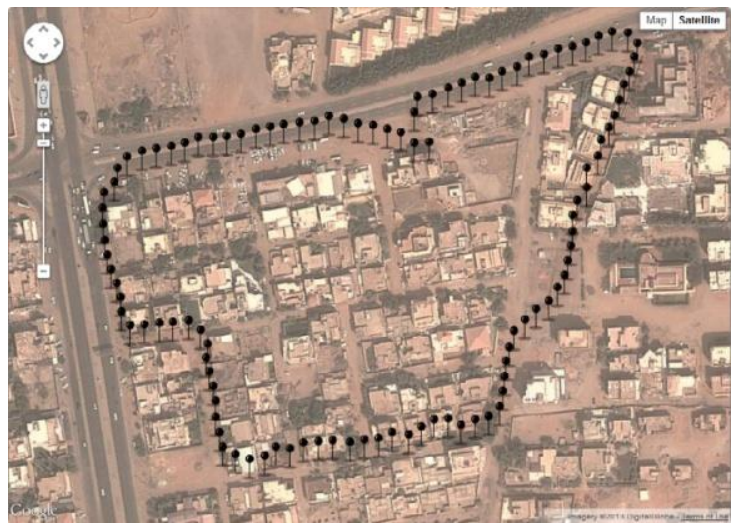

(3)

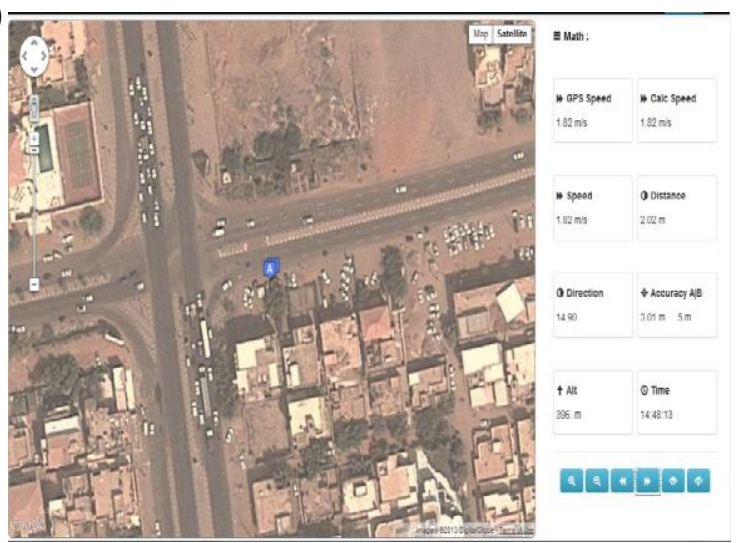

Fig 6: Google Earth Snapshot showing (1) transmitted GPS position and (2) corrected location of the tracked Smartphone.

\section{CONCLUSION AND FUTURE WORK}

This paper studies the real-time location tracking and control system that can track the target position at all time and places as required by the user. Furthermore, this paper developed an optimized localization system depending on the integration of GPS and GPRS/3G techniques. This paper can conclude that the obtained result have the accepted accuracy to be used in determining the location in a high accuracy which can reach to almost less than 5 meters. The system helps to save the expense for the use of GSM network services. It has the device installed with the user's target and Android application as in smart phones to communicate with the tracking device. The application on Smart Phone is compatible only with Android Application.

For the future works, this research realized that GPS has weak signal in the area which is full of tall buildings, while it has strong signal in the area where there aren't tall buildings or in open spaces.

The weak signal of GPS constitute a big problem in the tracking process, so to solve this problem this research are going to use Wi-Fi networks to determine the location of the target. 


\section{REFERENCES}

[1] Katina Michael, Andrew McNamee, MG Michael, "The Emerging Ethics of Humancentric GPS Tracking and Monitoring", Mobile Business, 2006. ICMB '06. International Conference on, pp. 1-10 , 2006.

[2] M. Zahaby, P. Gaonjur, and S. Farajian, "Location tracking in GPS using Kalman Filter through SMS", IEEE EUROCON 2009 (EUROCON '09), pp. 17071711,2009

[3] Peng Wang, Zhiwen Zhao, Chongbin $\mathrm{Xu}$, Zushun $\mathrm{Wu}$, and Yi Luo, "Design and implementation of the lowpower tracking system based on GPS-GPRS module", the 5th IEEE Conference on Industrial Electronics and Applications 2010 (ICIEA'2010), pp. 207-210, 2010.

[4] Sathiamoorthy Manoharan, "On GPS Tracking of Mobile Device", in Networking and Services, 2009. ICNS '09. Fifth International Conference on, pp. 1-4,2009.

[5] Cuiju Li, Zhizheng Zhou, Feng Yang, Shuming Jiang, and Liping Wang, "Design and Implementation of Modern Logistics Vehicles and Cargo Tracking Systems", International Seminar on Future BioMedical Information Engineering, 2008 (FBIE '08), pp. 411-414, 2008 .

[6] S. A. Hameed, O. Khalifa, M. Ershad, F. Zahudi, B. Sheyaa, and W. Asender, "Car monitoring, alerting and tracking model: Enhancement with mobility and database facilities", in Computer and Communication Engineering (ICCCE), 2010 International Conference on, 2010, pp. 15 .

[7] H.A. Rahim, R.B. Ahmad, A.S.M. Zain, and U.U. Sheikh, "Implementation and analysis of integration GSM/GPRS modem in a TMS320VC6713 digital signal processor for vehicle location", International Conference on Computer and Communication Engineering 2010 (ICCCE’2010), pp. 1-5, 2010

[8] Wei Hu, Tianzhou Chen, Qingsong Shi, Xueqing Lou , 2010 Smartphone Software Development Course Design Based on Android ,Computer and Information
Technology (CIT), 2010 IEEE 10th International Conference, $2180-2184$, DOI = 10.1109/CIT.2010.374

[9] Songphon Namkhun, Daranee Hormdee "Two-Way Semi-Offline Location Tracking and Control System via GSM" ITC-CSCC 2011, June 19-22, 2011, Gyeongju, Korea.

[10] Xun Li, Ortiz, P.J., Browne, J., Franklin, D., Oliver , J.Y., Geyer, R., Yuanyuan Zhou Chong, 2010 Smartphone Evolution and Reuse: Establishing a More Sustainable Model, IEEE 39 The International Conference , 13-16Sept. 2010, 476 - 484 DOI=10.1109/ICPPW.2010.70.

[11] ZACKS,(2011).Available;http://www.zacks.com/stock/n ews/35221/Smartphone+ Growth+to+Accelerate.

[12] Maoqiang Song, Wenkuo Xiong, Xiangling Fu, 2010 , Research on Architecture of Multimedia and Its Design Based on Android, Internet Technology and Applications, IEEE International Conference, 20-22 August 2010, 1 - 4 , DOI =10.1109/ITAPP.2010.556665

[13] Kuzmanovic, N., Maruna, T., Savic, M., Miljkovic , G., Isailovic, D., 2010, Google's android as an application environment for DTV decoder system , Consumer Electronics(ISCE), IEEE 14 Th International Symposium, 7-10 June 2010, $1-5$, DOI $=101109$ /ISCE.2010.5522728

[14] AndroidDevelopers,(2011).Avalable;http://developer.and roid.com/guide/basics/what-isandroid.html.

[15] Kumar Maji, A., Kangli Hao, Sultana, S., Bagchi , S., Characterizing Failures in Mobile OSes: A Case Study with Android and Symbian, Software Reliability Engineering(ISSRE), IEEE $21 \mathrm{St}$ International Symposium, San Jose, CA, 1-4 Nov. 2010 , 249 - 258 , DOI= 10.1109/ISSRE.2010.45

[16] Thuong Le-Tien and Vu Phung-The, "Routing and Tracking System for Mobile Vehicles in Large Area", the $5^{\text {th }}$ IEEE International Symposium on Electronic Design, Test and Application, 2010 (DELTA '2010), pp. 297300,2010 